\title{
Assessing Health Care Burden in Glaucoma Patients with and Without Physical or Mental Comorbidities
}

\author{
Michael Serbin, PharmD, MS; Beth Devine, PharmD, PhD, MBA; \\ Joanna Campbell, PhD; and Anirban Basu, PhD
}

\begin{abstract}
BACKGROUND: Glaucoma is a collection of eye diseases that damage the eye's optic nerve resulting in vision loss and blindness. Treatment for glaucoma is primarily pharmacologic; however, studies have shown patients have difficulty adhering to topical regimens. The reasons for potentially poor adherence are numerous, including influence from a myriad of either physical or mental comorbid conditions faced by many glaucoma patients. Neither adherence nor associated outcomes have been estimated in these 2 groups of glaucoma patients.
\end{abstract}

OBJECTIVES: To (a) characterize glaucoma patients with and without select physical or mental comorbidities and (b) estimate differences between the 2 groups for 3 types of outcomes: health care resource use (HCRU; officebased/outpatient-based provider visits, emergency room visits, inpatient stays, home health provider days, prescription fills); health care expenditures; and health-related quality of life ( $\mathrm{HRQOL})$ as measured by the physical and mental component scores of the Short Form-12.

METHODS: We used first-year data from each glaucoma patient's 2-year panel survey in the Medical Expenditure Panel Survey (MEPS) database, 2003-2014. Two groups were created using ICD-9-CM codes collected by MEPS to compare glaucoma patients with and without at least 1 selected physical or mental comorbid condition. Between-group comparisons in the outcomes of interest (HCRU, expenditure, HRQoL) were estimated using multivariable regression analyses while adjusting for socio-demographic and clinical characteristics at baseline.

RESULTS: We identified 2,928 unique glaucoma patients during the 11 years of collected data, including 1,539 (53\%) who had at least 1 physical or mental comorbid condition of interest. Comparing those with at least 1 select physical or mental comorbidity to those without $(n=1,389)$, unadjusted HCRU and expenditures were greater in patients with a physical or mental comorbidity (all $P<0.05$ ). After adjustment, significant associations with increased HCRU remained for office-based provider visits and home health provider days (each $P<0.01$ ). Average total expenditures were $\$ 12,324$ in those with comorbidities and $\$ 8,590$ for those without. HRQOL (unadjusted and adjusted) was lower in those with a physical or mental comorbid condition (all $P<0.05$ ).

CONCLUSIONS: Some differences in HCRU and expenditures were accounted for by differences in baseline characteristics between those with and those without 1 or more physical or mental comorbid conditions, but differences remained after adjustment. Results suggest that glaucoma patients with physical and mental comorbidities may experience greater HCRU and associated expenditures, and lower HRQoL, when compared with glaucoma patients without these comorbidities With this knowledge, future work may include estimating the effect of the number of these comorbid conditions on each of the 3 types of outcomes.

J Manag Care Spec Pharm. 2020;26(3):325-31

Copyright $\odot 2020$, Academy of Managed Care Pharmacy. All rights reserved.

\section{What is already known about this subject}

Glaucoma is a collection of eye diseases that damage the eye's optic nerve, resulting in vision loss and is one of the world's leading causes of blindness.

The burden of disease on the health care system is large, responsible for billions of dollars in estimated annual direct medical costs in the United States.

Although there are readily available topical pharmacologic (drop) treatments for glaucoma, these drugs are often used multiple times per day, which can lead to potentially poor adherence.

\section{What this study adds}

An exploratory analysis was conducted to estimate the effect of physical and mental comorbidities on health care resource use (HCRU), associated expenditures, and health-related quality of life (HRQOL)

Results suggest that glaucoma patients with physical and mental comorbidities may experience greater HCRU and associated expenditures, and lower HRQoL, than glaucoma patients without these comorbidities, highlighting the potential burden of these conditions.

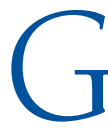
laucoma is a collection of eye diseases that damage the eye's optic nerve resulting in vision loss and blindness. ${ }^{1}$ Glaucoma is the second leading cause of blindness in the world and is responsible for more than 120,000 prevalent cases of blindness in the United States. ${ }^{2}$ Moreover, the disease exerts a heavy cost burden, with an estimate of more than $\$ 2.9$ billion in direct medical costs yearly to the United States. ${ }^{3}$ Vision loss in glaucoma develops most often through increased ocular pressure, the most significant modifiable risk factor, over time. ${ }^{1}$ Primary open-angle glaucoma, the most commonly occurring subset of the disease, is categorized as a chronic, noncurable disease managed with a number of treatment modalities including medication and surgery. ${ }^{4}$

Current treatment for glaucoma is primarily pharmacologic. Mainstays of the current treatment paradigm include alpha agonists and beta blockers, which came to market in the 1950s and 1960s, and topical prostaglandins, beginning in the late 1990s, that allowed for multiple lines of topical medications (drop treatments). ${ }^{4}$ Despite being the first-line choice, 
the potential exists for poor adherence to drop treatments. ${ }^{4}$ Glaucoma medication nonadherence has been documented to have significant effects on vision-related quality of life. ${ }^{5}$ Innovative nondrop treatments, including laser procedures and minimally invasive glaucoma surgeries (MIGs) involving stents and shunts, are treatment modalities that may improve adherence and persistence. ${ }^{6-8}$ Patients who struggle with drop treatments due to their comorbidities could potentially benefit from these innovative treatment modalities.

The objectives of this study were to describe patient characteristics and explore differences in health care resource use (HCRU), expenditures, and health-related quality of life (HRQoL) in glaucoma patients who do and do not have at least 1 select physical or mental comorbidity (SPMC). To our knowledge, we are the first to conduct such analysis. Characterizing these patients may assist in understanding the unmet need for these innovative treatment modalities.

\section{Methods}

\section{Medical Expenditure Panel Survey}

We used data from the Medical Expenditure Panel Survey (MEPS). MEPS is a nationally representative subsample of the National Health Interview Survey, which is a multipurpose and continuously conducted survey of the U.S. noninstitutionalized civilian population. MEPS oversamples certain policy-relevant subgroups of the population including low-income households, whereas the National Health Interview Survey oversamples black, Hispanic, and Asian individuals. ${ }^{9}$ MEPS data are collected in 2-year longitudinal panels over 5 rounds, with overlapping panels in each calendar year.

For this study, we used a cross-sectional approach using yearly calendar files from 2003 to 2014, the last year of finished MEPS files available in June 2017. Data collected in the first year of each 2-year MEPS panel were used for each patient to prevent bias arising from including the same patient twice in the analysis in 2 separate years. MEPS is considered a public dataset; the data are deidentified and Health Insurance Portability and Accountability Act compliant. Therefore, the study did not meet the definition of human subject research and did not require approval by the University of Washington Institutional Review Board

We identified the relevant International Classification of Diseases, Ninth Revision, Clinical Modification (ICD-9-CM) codes and mapped those to the codes used in the MEPS database. Using this MEPS terminology, we were then able to identify patients diagnosed with any diagnosis of glaucoma and to identify the presence or absence of 1 or more physical or mental comorbidities that could affect a patient's suitability for glaucoma drop treatments (Appendix A, available in online article). Decisions about which comorbidities to include were made by consulting ophthalmologists who specialize in the treatment of glaucoma. The purpose of dividing the glaucoma patient population into those with versus without these comorbid conditions was to characterize the extent to which glaucoma patients who had 1 of these comorbidities would incur greater HCRU and expenditures and experience lower HRQoL than glaucoma patients without 1 or more of these comorbidities.

Although we were unable to directly test this hypothesis with the available data, we postulated that glaucoma patients with comorbidities could be less adherent to drop treatment, which could independently affect HCRU, expenditures, or HRQoL. Once identified using the MEPS yearly medical conditions file, the patient identification number was used to extract demographic (age, sex, race, years of education, insurance, employment status, and salary) and outcome data (HCRU, expenditures, HRQoL) using the MEPS full-year consolidated data files To remove as much influence in the SPMC population caused by the underlying SPMC, we also added 1 MEPSdefined covariate to represent serious cognitive disability and 1 MEPS-defined variable to represent difficulty in walking or climbing stairs. (Appendix B, available in online article).

Finally, the Charlson Comorbidity Index (CCI) score was calculated-using the D'Hoore method (range 0-30) with the MEPS and ICD-9-CM codes ${ }^{10}$ - and used as a covariate.

\section{Health Care Resource Utilization and Health Expenditures}

HCRU outcomes of interest include provider visits (office based and outpatient based), emergency room visits, hospital inpatient admissions, home health provider days, and prescription drug fills.

Analogous MEPS expenditure variables correspond to HCRU outcomes of interest, except for home health care and inpatient visit expenditures. For these 2 outcomes, individual components of expenditures in each category were summed to a total expenditure, as there was no predefined MEPS total. The Personal Consumption Expenditure Health Price Indices were used to adjust each expenditure to inflation-adjusted 2014 U.S. dollars. ${ }^{11}$

\section{Health-Related Quality of Life}

MEPS collects HRQoL data using the 12-item Short Form Survey (SF-12), which produces a physical component score (PCS) and a mental component score (MCS). The PCS and MCS have a national mean in the U.S. population of 50 and a standard deviation of 10, meaning those who score above 50 have better than average health than the typical person in the general U.S. population (age is not held constant) and those below 50 have worse than average health. ${ }^{12}$ Each 1-point difference above or below 50, in either component, corresponds to one tenth of 1 standard deviation.

\section{Primary and Secondary Analyses}

In our primary analysis, we compared HCRU, expenditures, and HRQoL in glaucoma patients who do and do not have at 


\section{TABLE 1 Patient Demographics and Disability Status}

\begin{tabular}{|c|c|c|c|c|}
\hline \multirow[b]{2}{*}{$\mathrm{N}(\%)$} & Total & Without SPMC & \multicolumn{2}{|c|}{ With SPMC } \\
\hline & 2,928 & $1,389 \quad(47.4)$ & 1,539 & $(52.6)$ \\
\hline Age (SD, range) & $65.09 \quad(15.84,1-85)$ & $64.39 \quad(16.31,1-85)$ & 65.73 & $(15.37,3-85)^{\mathrm{a}}$ \\
\hline Female, n (\%) & $1,708 \quad(58.3)$ & $749 \quad(53.9)$ & 959 & $(62.3)^{\mathrm{b}}$ \\
\hline \multicolumn{5}{|l|}{ Race, n (\%) } \\
\hline White & $1,971 \quad(67.3)$ & $914 \quad(65.8)$ & 1,057 & $(68.7)$ \\
\hline Black & $735 \quad(25.1)$ & $359 \quad(25.8)$ & 376 & $(24.4)$ \\
\hline Asian & $156(5.3)$ & $88 \quad(6.3)$ & 68 & $(4.4)$ \\
\hline Other/multiple races reported & $66 \quad(2.3)$ & $28 \quad(2.0)$ & 38 & $(2.5)$ \\
\hline \multicolumn{5}{|l|}{ Education, n (\%) } \\
\hline No education & $21 \quad(0.7)$ & $12 \quad(0.9)$ & 9 & $(0.6)$ \\
\hline High school or less & $1,243 \quad(42.5)$ & $630 \quad(45.4)$ & 613 & $(39.8)$ \\
\hline $1-3$ years of college & 368 (12.6) & $183(13.2)$ & 185 & (12.3) \\
\hline 4 years of college & $250 \quad(8.5)$ & $124 \quad(8.9)$ & 126 & $(8.2)$ \\
\hline $5+$ years of college & $216 \quad(7.4)$ & $111 \quad(8.0)$ & 105 & $(6.8)$ \\
\hline Unreported/not ascertained & $830 \quad(28.3)$ & $329 \quad(23.7)$ & 501 & $(32.6)$ \\
\hline \multicolumn{5}{|l|}{ Insurance, $\mathrm{n}(\%)$} \\
\hline Private insurance & $1,608 \quad(54.9)$ & $777 \quad(56.0)$ & 831 & $(54.0)^{\mathrm{b}}$ \\
\hline Public insurance & $1,171 \quad(40.0)$ & $525 \quad(37.8)$ & 646 & $(42.0)^{\mathrm{b}}$ \\
\hline Uninsured & $149(5.1)$ & $87(6.3)$ & 62 & $(4.0)^{\mathrm{b}}$ \\
\hline \multicolumn{5}{|l|}{ Employment, n (\%) } \\
\hline Employed/job to return to & 909 (3.1) & $480 \quad(34.6)$ & 429 & $(27.9)^{\mathrm{b}}$ \\
\hline Unemployed & $1,952 \quad(66.7)$ & $874(62.9)$ & 1,078 & $(70.0)^{\mathrm{b}}$ \\
\hline Unreported/not ascertained & $67(2.3)$ & $35(2.5)$ & 32 & $(2.1)^{\mathrm{b}}$ \\
\hline Mean wage, USD (SD) & $13,848 \quad(27,664)$ & $15,159 \quad(28,712)$ & 12,666 & $(26,637)^{\mathrm{a}}$ \\
\hline Serious cognitive difficulties, $\mathrm{n}(\%)$ & $355(12.1)$ & $91 \quad(6.6)$ & 264 & $(17.2)^{\mathrm{b}}$ \\
\hline Serious difficulty walking/climbing stairs, n (\%) & $966(33.0)$ & $322 \quad(23.2)$ & 644 & $(41.8)^{\mathrm{b}}$ \\
\hline Charlson Comorbidity Index, on a score of 0-30 (SD) & $3.91 \quad(3.92)$ & $2.28(2.84)$ & 5.38 & $(4.17)^{\mathrm{b}}$ \\
\hline
\end{tabular}

least 1 SPMC. In our secondary analyses, we evaluate these same 3 outcomes in subgroups of glaucoma patients with only 1 or more physical comorbidities versus no physical comorbidity and only 1 or more mental comorbidities versus no mental comorbidity.

\section{Statistical Analysis}

In glaucoma patients, differences in demographic and disability characteristics between those with and without SPMC subgroups were tested for significance using chi-square tests for categorical outcomes and simple linear regressions for continuous outcomes. Comparisons between the 2 groups in the HCRU outcome were evaluated using negative binomial regression models; negative binomial models are appropriately used when count outcomes are of interest, yet they are heavily right skewed. Incident rate ratios (IRRs) were estimated using both unadjusted and adjusted models, the latter adjusting for differences in the 2 groups in demographic and disability variables. Covariate-adjusted comparisons in expenditures between the
2 groups were estimated using extended estimating equations (EEE) ${ }^{13}$ which improved model fit over the more traditionally used generalized linear model with a gamma family and log link. For each model, the method of recycled predictions was used to estimate differences in expenditures. For 1 outcome, outpatient-based provider expenditures, no model with the majority of covariates would converge using EEE. Therefore, the alternative 2-part model, first part logit, second part loglink, gamma family generalized linear regression, was used instead. Differences in HRQoL were tested using ordinary least squares regression models, both unadjusted and adjusted for potential confounding covariates. The same analyses were used to evaluate each secondary outcome. Analyses were conducted primarily using $\mathrm{R}$ version 3.3.0 statistical software; STATA 13-IC version 13.1 (StataCorp, College Station, TX) was used in regressions that estimated expenditure outcomes, as programming code for extended estimating equations was only available in STATA. All comparisons were performed using a 2-tailed alpha level of 0.05 . 
TABLE 2 Health Care Resource Utilization

\begin{tabular}{|c|c|c|c|c|c|}
\hline Outcome & $\begin{array}{c}\text { Without SPMC } \\
\text { (Mean) }\end{array}$ & $\begin{array}{c}\text { With SPMC } \\
\text { (Mean) }\end{array}$ & $\begin{array}{l}\text { Unadjusted } \\
\text { Difference }\end{array}$ & $\begin{array}{l}\text { Unadjusted IRR } \\
\quad(95 \% \mathrm{CI})\end{array}$ & $\begin{array}{l}\text { Adjusted }^{\text {a }} \text { IRR } \\
(95 \% \mathrm{CI})\end{array}$ \\
\hline Office-based provider visits (OBTOTV) & 9.54 & 13.69 & 4.15 & $\begin{array}{c}1.434 \\
(1.33-1.55)^{\mathrm{b}}\end{array}$ & $\begin{array}{c}1.11 \\
(1.03-1.20)^{\mathrm{b}}\end{array}$ \\
\hline Outpatient-based provider visits (OPTOTV) & 0.98 & 1.51 & 0.53 & $\begin{array}{c}1.54 \\
(1.28-1.85)^{\mathrm{b}}\end{array}$ & $\begin{array}{c}1.17 \\
(0.97-1.42)\end{array}$ \\
\hline Emergency room visits (ERTOT) & 0.25 & 0.38 & 0.14 & $\begin{array}{c}1.55 \\
(1.29-1.87)^{\mathrm{b}}\end{array}$ & $\begin{array}{c}1.04 \\
(0.852-1.27)\end{array}$ \\
\hline Inpatient hospital stays (IPDIS) & 0.16 & 0.26 & 0.11 & $\begin{array}{c}1.69 \\
(1.36-2.10)^{b}\end{array}$ & $\begin{array}{c}1.06 \\
(0.84-1.34) \\
\end{array}$ \\
\hline Home health provider days (HHTOTD) & 3.87 & 12.35 & 8.448 & $\begin{array}{c}3.20 \\
(2.14-4.79)^{\mathrm{b}}\end{array}$ & $\begin{array}{c}1.91 \\
(1.25-2.90)^{\mathrm{b}}\end{array}$ \\
\hline Prescription fills (RXTOT) & 27.07 & 38.51 & 11.44 & $\begin{array}{c}1.42 \\
(1.32-1.54)^{b}\end{array}$ & $\begin{array}{c}1.01 \\
(0.94-1.09)\end{array}$ \\
\hline
\end{tabular}

aAdjusting for age, gender, race, insurance, employment status, wage, physical limitation, cognitive limitation, and CCI.

${ }^{b} P<0.01$, with versus without those with SPMC.

$C C I=$ Charlson Comorbidity Index; $C I=$ confidence interval; $I R R=$ incident rate ratios; $S P M C=$ select physical or mental comorbidity.

\section{Results}

\section{Primary Analysis}

Over the 11-year period between 2003 and 2014, 2,928 unique glaucoma patients were identified in the MEPS database. Of the 2,928 who had a MEPS code for glaucoma diagnosis, 1,539 (52.6\%) also had an ICD-9-CM diagnosis for at least 1 physical or mental comorbidity (Table 1). Significant differences between the 2 subgroups were seen in age $(P=0.023)$, gender $(P<0.001)$, insurance coverage $(P=0.004)$, current employment $(P<0.001)$, average wages $(P<0.001)$, and both disability variables (serious cognitive disability and serious difficulty walking [each $P<0.001]$ ). The with-SPMC group was older, more likely female, less likely to be employed, and more likely to have limiting cognitive or walking disabilities.

\section{Health Care Resource Utilization}

As expected, the unadjusted IRR between glaucoma patients with and without SPMCs was significant for each of the 6 HCRU outcomes (Table 2). For unadjusted IRRs, greater than $50 \%$ increases were seen in the SPMC group in outpatient provider visits, emergency room visits, hospital stays, and home health care days. However, after adjusting for covariates, only differences in office-based provider visits, (adjusted IRR [adjIRR $]=1.11,95 \%$ confidence interval $[\mathrm{CI}]=1.03-1.20$ ), and home health provider days, (adjIRR $=1.91,95 \% \mathrm{CI}=1.25-2.90)$, remained significant. A 90\% increase, after adjustment, in home health provider days in glaucoma patients with an SPMC compared with those without suggests a much higher comparative resource use particularly for this outcome, whereas comparative differences in all other outcomes after adjustment were between $<1 \%$ (prescription fills) and 17\% (outpatient-based provider visits), with more usage in the with-SPMC subgroup.

\section{Expenditures}

All unadjusted expenditures were higher in the with-SPMC glaucoma group although no significant differences persisted after adjustment for covariates using regression models with extended estimating equations or the 2-part model (Table 3). Although outpatient-based provider, emergency room, inpatient hospital, and prescription drug expenditures all turned slightly negative in the adjusted analysis, the magnitudes were small and confidence intervals wide, with differences all including zero. In total, the expenditures of these 6 outcomes combined was, on average, $\$ 12,324$ in the SPMC group and $\$ 8,590$ for the without-SPMC group over 1 year of MEPS expenditure data.

\section{Health-Related Quality of Life}

Overall, glaucoma patients with SPMCs had worse unadjusted HRQoL in both the MCS and PCS of the SF-12 (each $P<0.001$; Table 4). On average, after adjustment, those in the SPMC group had an MCS score -1.28 points ( $95 \% \mathrm{CI}=-2.10$ to $-0.46, P<0.01)$ worse and a PCS -0.85 points $(95 \% \mathrm{CI}=-1.64$ to $-0.06 ; P<0.05)$ worse than those without an SPMC. As a whole, glaucoma patients in the combined cohort (SPMC and no SPMC) had an average MCS similar to the average population at 50.15 (SD 10.56), where mean SF-12 MCS is 50 and SD is 10 for the entire U.S. population. However, the full cohort had an average PCS was of 41.79 (SD 12.55), well below the population mean

\section{Secondary Analyses}

Physical Comorbidities. Separating the glaucoma patient group by the presence or absence of physical comorbidities alone revealed interesting findings. Home health provider days remained significantly different, with those who had a 


\section{TABLE 3 Expenditures}

\begin{tabular}{|c|c|c|c|c|}
\hline Outcome & $\begin{array}{c}\text { Without SPMC } \\
\text { (Mean) }\end{array}$ & $\begin{array}{c}\text { With SPMC } \\
\text { (Mean) }\end{array}$ & $\begin{array}{l}\text { Unadjusted } \\
\text { Difference }\end{array}$ & $\begin{array}{c}\text { Adjusted }{ }^{\mathrm{a}} \text { difference } \\
(95 \% \mathrm{CI})\end{array}$ \\
\hline Office-based provider expenditures (OBVEXP), USD & 2,047 & 2,688 & $641^{b}$ & $\begin{array}{c}77 \\
(-271.27-425.50) \\
\end{array}$ \\
\hline Outpatient-based provider expenditures (OPVEXP), USD & 439 & 497 & $58^{\mathrm{b}}$ & $\begin{array}{c}-3 \\
(-\$ 14-\$ 8)\end{array}$ \\
\hline Emergency room expenditures (ERTEXP), USD & 278 & 311 & $33^{b}$ & $\begin{array}{c}-42 \\
(-176.54-92.55) \\
\end{array}$ \\
\hline Inpatient hospital expenditures (IPTEXP), USD & 3,337 & 4,314 & $977^{\mathrm{b}}$ & $\begin{array}{c}-883 \\
(-2,230.72-463.38)\end{array}$ \\
\hline Home health expenditures (HHTEXP), USD & 406 & 1,014 & $608^{b}$ & $\begin{array}{c}116 \\
(-170.23-403.64)\end{array}$ \\
\hline Prescription drug expenditures (RXEXP), USD & 2,333 & 3,500 & $1,167^{b}$ & $\begin{array}{c}-3 \\
(-326.53-319.99) \\
\end{array}$ \\
\hline \multicolumn{5}{|c|}{$\begin{array}{l}\text { aAdjusting for age, gender, race, insurance, employment status, wage, physical limitation, cognitive limitation, and CCI (each outcome used a different set } \\
\text { needed because of limitations in attaining model convergence). See Appendix B. } \\
\text { bP }<0.01 \text {, with versus without SPMC. } \\
C C I=\text { Charlson Comorbidity Index; CI= confidence interval; IRR=incident rate ratios; SPMC= select physical or mental comorbidity; USD =U.S. dollars. }\end{array}$} \\
\hline
\end{tabular}

physical comorbidity more likely to use this health care resource $(\operatorname{adjIRR}=1.87,95 \% \mathrm{CI}=1.67-2.09 ; P<0.01)$. Unlike the larger primary analysis, home health provider expenditures were significantly different for this subgroup with an adjusted difference of $\$ 159$ (95\% CI $=\$ 7-\$ 312 ; P<0.05)$. Adjusted quality of life scores were now significant only for the PCS $(P<0.05)$, but not the MCS $(P=0.48)$.

Mental Comorbidities. In contrast with patients in the any physical comorbidity subgroup, analysis in patients in the any mental comorbidity subgroup revealed that adjusted HCRU differences were now statistically significant in all but 2 categories, including outpatient-based provider visits (adjIRR $=1.21$, 95\% $\mathrm{CI}=1.06-1.39$ ), emergency room visits (adjIRR $=1.22$, 95\% CI $=1.00-1.49$ ), home health provider days (adjIRR $=1.67,95 \%$ $\mathrm{CI}=1.46-1.91$ ), and prescription drug fills (adjIRR $=1.18$, 95\% CI=1.06-1.31), all $P<0.05$. Adjusted expenditure outcomes showed 2 significant values, first in home health provider expenditures (adjusted difference $=\$ 258,95 \% \mathrm{CI}=\$ 9-\$ 506$; $P<0.05)$ and second in prescription drug expenditures (adjusted difference $=\$ 429,95 \% \mathrm{CI}=\$ 44-\$ 813 ; P<0.05$ ) with those glaucoma patients with a mental comorbidity having higher yearly expenditures after covariate adjustment. The MCS after adjustment became a 3.36-point difference between those glaucoma patients with and without mental comorbidities; this more than doubled the adjusted difference in the primary analysis. The PCS difference for the mental comorbidity subgroup was not significantly different from the nonmental comorbidity glaucoma population $(P=0.816)$ following the same pattern as the physical comorbidity population not showing significant differences in MCS component score.

\section{Discussion}

Using the MEPS database, a representative sample of the U.S. civilian population, this cross-sectional study evaluated 11 years of the most recently collected MEPS data to assess characteristics, HCRU, expenditures, and HRQoL in the cohort of glaucoma patients with an SPMC compared with a cohort without. Demographic and disability characteristics were statistically different between the 2 groups, with SPMC patients on average older, female, less likely to be employed, and more likely to have limiting disabilities. As anticipated, glaucoma patients with an SPMC were more likely to use more health care resources, have higher expenditures, and have decreased HRQoL as measured by the SF-12. Because of the clear differences in baseline characteristics between the 2 groups, adjustments were made for baseline age, sex, race, insurance status, employment, wages, cognitive and walking disability, and CCI score. After adjustment, home health provider days and officebased provider visits remained significant; the other components of HCRU did not, nor did differences in expenditures. However, major differences in HRQoL (PCS and MCS) were apparent between these 2 groups even after adjustment.

In secondary analyses of each component of SPMC (only physical comorbid conditions and only mental comorbid conditions), additional significant differences were noted, even after adjustment. When comparing those with a physical comorbidity to those without, we found that home health provider days and associated expenditures were higher and the PCS of the SF-12 lower. When comparing those with a mental comorbidity to those without, we observed that outpatient visits, emergency room visits, home health provider days, and prescription fills were all significantly higher, as were the associated expenditures for home health provider days and 
TABLE 4 Health-Related Quality of Life Using SF-12 Mental and Physical Component Scores

\begin{tabular}{|c|c|c|c|c|c|}
\hline SF-12 Component & $\begin{array}{l}\text { Total } \\
\text { Mean } \\
\text { (SD) }\end{array}$ & $\begin{array}{l}\text { Without SPMC } \\
\text { Mean } \\
\text { (SD) }\end{array}$ & $\begin{array}{l}\text { With SPMC } \\
\text { Mean } \\
\text { (SD) }\end{array}$ & $\begin{array}{c}\text { Difference } \\
\text { (Unadjusted) } \\
(95 \% \mathrm{CI})\end{array}$ & $\begin{array}{c}\text { Difference } \\
\text { (Adjusted a) } \\
(95 \% \mathrm{CI}) \\
\end{array}$ \\
\hline Mental component score & $\begin{array}{l}50.17 \\
(10.56)\end{array}$ & $\begin{array}{l}51.79 \\
(9.512) \\
\end{array}$ & $\begin{array}{l}48.74 \\
(11.22)\end{array}$ & $\begin{array}{c}-3.049 \\
(-3.833 \text { to }-2.266)^{\mathrm{b}}\end{array}$ & $\begin{array}{c}-1.278 \\
(-2.099 \text { to }-0.458)^{b}\end{array}$ \\
\hline Physical component score & $\begin{array}{c}41.79 \\
(12.55)\end{array}$ & $\begin{array}{c}44.67 \\
(11.66)\end{array}$ & $\begin{array}{c}39.22 \\
(12.76)\end{array}$ & $\begin{array}{c}-5.458 \\
(-6.381 \text { to }-4.534)^{b}\end{array}$ & $\begin{array}{c}-0.849 \\
(-1.640 \text { to }-0.058)^{c}\end{array}$ \\
\hline
\end{tabular}

aAjusting for age, gender, race, insurance, employment status, wage, physical limitation, cognitive limitation, and CCI.

${ }^{b} P<0.01$, with versus without SPMC

${ }^{c} P<0.05$.

$C I=$ confidence interval; SF-12 =12-item short form; SPMC=select physical or mental comorbidity; USD=U.S. dollars

prescription fills. The MCS of the SF-12 was lower in those with a mental comorbidity.

Although regression analyses and adjustments for covariates were conducted for all outcomes of interest, the primary focus of this study was not addressing whether glaucoma patients with an SPMC use more resources, as would be expected. Rather, our focus was to quantify this difference in HCRU, expenditures, and HRQoL, as characterizing these glaucoma patients who have an SPMC is important in understanding the unmet need for alternatives to current therapy.

Other investigators have estimated HCRU, costs, and HRQoL in glaucoma patients, although no studies to date have looked at the subset of glaucoma patients with physical or mental comorbidities. ${ }^{14-16}$ Separately, a rationale for additive comorbid conditions affecting medication adherence was investigated by Rolnick et al. (2013), where the addition of a greater number of comorbid conditions was associated with worse medication adherence. Rolnick's study, which focused on 8 disease states, did not include glaucoma. ${ }^{17}$

\section{Limitations}

This study has a few limitations to consider. First, despite its strength and usefulness, MEPS is fundamentally a survey dataset, which comes with inherent biases, such as oversimplification of answers and recall bias. Second, the aggregation of ICD-9-CM codes in MEPS does not allow focus on primary open-angle glaucoma. This results in a combination of glaucoma diagnoses, including acute conditions.

Third, the makeup of the SPMC group, which uses a large number of heterogeneous physical and mental comorbidities, precludes a more granular understanding of the effect of each individual comorbidity. Further subgrouping by each individual comorbid condition would have led to populations too small to conduct meaningful statistical inference. Moreover, due to issues with model convergence, we were unable to use the same set of covariates in all regression analyses. Fourth, because MEPS does not provide granularity about type of glaucoma intervention (topical, surgical, laser), we were unable to assess whether those receiving topical treatment fared worse than those receiving other types of interventions.

Fifth, although other authors have found that comorbidities are associated with worse medication adherence in other disease states and separately that worse adherence to glaucoma medications is associated with lower vision-related QoL, ${ }^{17}$ limitations in our dataset prevented us from assessing adherence as a mediator in our analysis. That is, without information about adherence, we were unable to evaluate our hypothesis that comorbidities affect adherence to glaucoma treatments which, in turn, affects outcomes. It could be that the comorbidities themselves contribute to worse outcomes, regardless of adherence to glaucoma treatments.

Finally, as we limited our sample to only those who had a diagnosis of glaucoma, we were unable to assess the interaction between the presence of glaucoma and the comorbid conditions.

Our exploratory analysis into the effect of physical and mental comorbidities on outcomes in glaucoma patients will springboard to future work. Specifically, a database that distinguishes type of treatment (drops or surgery/laser), and wherein adherence can be estimated, will better enable us to evaluate the hypothesis that comorbidities reduce adherence which, in turn, can contribute to poorer outcomes. A claims database would lend nicely to this. In this same context, exploring how the number of comorbid conditions affects outcomes will further enhance our understanding of the role of innovative therapies.

\section{Conclusions}

The results of our nationally representative sample of glaucoma patients represented in MEPS data suggest that patients with physical or mental comorbidities experience higher HCRU and expenditures and lower HRQoL when compared with those without these comorbidities. This study quantifies the excess burden of select physical or mental comorbidities in patients with glaucoma. 


\section{Authors}

MICHAEL SERBIN, PharmD, MS, The Comparative Health Outcomes, Policy, and Economics (CHOICE) Institute, Department of Pharmacy and the Departments of Health Services and Economics, University of Washington, Seattle; Allergan, Irvine, California; and Neurocrine Biosciences, San Diego, California. BETH DEVINE, PharmD, PhD, MBA, and ANIRBAN BASU, PhD, The Comparative Health Outcomes, Policy, and Economics (CHOICE) Institute, Department of Pharmacy and the Departments of Health Services and Economics, University of Washington, Seattle. JOANNA CAMPBELL, PhD, Allergan, Irvine, California.

AUTHOR CORRESPONDENCE: Beth Devine, PhD, PharmD, MBA, Professor, The CHOICE Institute, University of Washington Department of Pharmacy, Box 357630, Seattle, WA 98195-7630. Tel.: 206.221.5760; E-mail: bdevine@uw.edu.

\section{DISCLOSURES}

This study received funding support from Allergan. During the time this work was conducted, Serbin was a postdoctoral fellow who was supported by a training grant from Allergan to the University of Washington. Campbell is an employee of Allergan. Serbin, Devine, and Basu each have nothing to disclose.

This study was presented as a poster at the International Society for Pharmacoeconomics and Outcomes Research Meeting; May 20-24, 2017; Boston, MA.

\section{REFERENCES}

1. National Eye Institute. Glaucoma. Updated July 29, 2019. Available at: https:// nei.nih.gov/health/glaucoma/glaucoma_facts. Accessed February 4, 2020.

2. Glaucoma Research Foundation. Glaucoma facts and stats. Reviewed October 29, 2017. Available at: https://www.glaucoma.org/glaucoma/glaucoma-facts-and-stats.php. Accessed February 4, 2020.

3. Rein DB, Zhang P, Wirth KE, et al. The economic burden of major adult visual disorders in the United States. Arch Ophthalmol. 2006;124(12):1754-60.

4. Realini T. A history of glaucoma pharmacology. Optometry Vis Sci. 2011; 88(1):36-38.
5. Thompson A C, Woolson S, Olsen MK, Danus S, Bosworth HB, Muir KW. Relationship between electronically measured medication adherence and vision-related quality of life in a cohort of patients with open-angle glaucoma. BMJ Open Ophthalmol. 2008;3(1):e000114.

6. Manasses DT, Au L. The new era of glaucoma micro-stent surgery. Ophthalmol Ther. 2016;5(2);135-46.

7. Malvankar-Mehta MS, Chen YN, Iordanous Y, Wang WW, Costella J, Hutnik CM. iStent as a solo procedure for glaucoma patients: a systematic review and meta-analysis. Plos One. 2015;10(5):e0128146.

8. Stein JD, Kim DD, Peck WW, Giannetti SM, Hutton DW. Cost-effectiveness of medications compared with laser trabeculoplasty in patients with newly diagnosed open-angle glaucoma. Arch Ophthalmol. 2012;130(4):497-505.

9. Agency for Healthcare Research and Quality. Medical Expenditure Panel Survey background. Revised April 22, 2019. Available at: https://meps.ahrq. gov/mepsweb/about_meps/survey_back.jsp. Accessed February 4, 2020.

10. D'Hoore W, Bouckaert A, Tilquin C. Practical considerations on the use of the Charlson Comorbidity Index with administrative data bases. J Clin Epidemiol. 1996;49(12):1429-33.

11. Agency for Healthcare Research and Quality. Using appropriate price indices for analyses of health care expenditures or income across multiple years. Updated February 7, 2019. Available at: https://meps.ahrq.gov/about_ meps/Price_Index.shtml. Accessed February 4, 2020.

12. U.S. Bureau of Labor Statistics. National longitudinal survey of youth 1979: NLSY79 appendix 19: SF-12 health scale scoring. 2017-2018. Available at: https://www.nlsinfo.org/content/cohorts/nlsy79/other-documentation/ codebook-supplement/nlsy79-appendix-19-sf-12-health-scale. Accessed February 4, 2020.

13. Basu A, Rathouz PJ. Estimating marginal and incremental effects on health outcomes using flexible link and variance function models. Biostatistics. 2005;6(1):93-109.

14. Stein JD, Niziol LM, Musch DC, et al. Longitudinal trends in resource use in an incident cohort of open-angle glaucoma patients: resource use in open-angle glaucoma. Am J Ophthalmol. 2012;154(3):452-59.e2.

15. Prager AJ, Liebmann JM, Cioffi GA, Blumberg DM. Self-reported function, health resource use, and total health care costs among Medicare beneficiaries with glaucoma. JAMA Ophthalmol. 2016;134(4):357.

16. Muratov S, Podbielski DW, Kennedy K, et al. Preference-based glaucoma-specific health-related quality of life instrument: development of the health utility for glaucoma. J Glaucoma. 2018;27(7):585-91.

17. Rolnick SJ, Pawloski PA, Hedblom BD, Asche SE, Bruzek RJ. Patient characteristics associated with medication adherence. Clin Medicine Res. 2013;11(2):54-65. 
APPENDIX A ICD-9-CM and MEPS Codes for Select Physical and Mental Comorbidities

Subgroup

ICD-9-CM Codes

MEPS Codes

Select physical comorbidities

Gout

Tics

Movement disorders

Hereditary \& degenerative disease of the CNS

(PD, ALS, other movement disorders)

Multiple sclerosis, paralysis, epilepsy, cerebral palsy

Late effects of cerebrovascular disease

Mononeuritis of upper limb

Hereditary \& idiopathic peripheral neuropathy

Inflammatory and toxic neuropathy

Myoneuronal disorders, muscular dystrophy

SLE

Polymyositis

Crystal arthropathies

RA

Joint derangement

\begin{tabular}{|l|l|}
\hline $274 . x x$ & 27 \\
\hline $307.2 \mathrm{x}$ & $307.3 \mathrm{x}$ \\
\hline $332 . x \mathrm{x}, 333,334,335 . \mathrm{xx}$ & 33 \\
\hline $340 . x x-342 . x x, 344.0,344.2,344.4,345$ \\
\hline
\end{tabular}

438.2, 438.3, 438.5

354.xx (except 354.2 and 354.3)

356.xx

357

358.xx-359.xx

710.0

710.4

712.x1-712.x4

714.xx

715.x1-715.x4

718.x1-718.x4

274

307 is special symptom NEC

N/A

331 cerebral degeneration, 332 Parkinson, 333 EPS

Joint disorder

719.x1-719.x4

Reduction deformities of upper limb

$755.2 \mathrm{x}$

340 multiple sclerosis, 343 infantile cerebral palsy, 345 epilepsy, 344 other paralytic syndromes

436 CVA, 437 other cerebrovascular disease

354 mononeuritis upper limp, 355 mononeuritis leg 357 Neuropathy

N/A

359 muscular dystrophies

710 connective tissue disorders

710 connective tissue disorders

N/A

714 other inflammatory polyarthropathy

$715 \mathrm{OA}$

717 internal joint derangement, 718 other joint derangement

719 Joint disorder

754 congenital musculoskeletal deformities, 755 other congenital limb anomalies, 756 other musculoskeletal anomalies

Down, Patau, and Edward syndrome

758.0-758.2

More than 1 physical type

2 or more physical (but no mental/CNS)

Select mental comorbidities

Dementia and psychoses

295 schizophrenic disorder, 296 affective

psychoses, 298 other nonorganic psychoses,

299 pervasive development disorders

Other cerebral degenerations (include AD)

Dissociative amnesia

Alcohol dependence

Drug dependence

Physiological malfunction arising from mental

factors (musculoskeletal)

Nonpsychotic mental disorder due to brain damage

Intellectual disability

Anoxic brain damage

Encephalopathy

Other CNS disorders

\begin{tabular}{|l|l|}
\hline $290 . x x-299 . x x$ & 294, other organic psych condition, \\
& \\
\hline $331.0-2$ & 331 cerebral degeneration \\
\hline 300.12 & 300 neurotic disorders 301 personality disorders \\
\hline $303 . x x$ & 303 alcohol dependence syndrome \\
\hline $304 . x x$ & 304 drug dependence \\
\hline 306.0 & N/A \\
\hline $310 . x x$ & N/A \\
\hline $317 . x x-319 . x x$ & 315 specific developmental delays \\
\hline 348.1 & 348 other brain conditions \\
\hline $348.3 x, 291.2,572.2,437.2,349.82$ & 437 other cerebrovascular disease, 349 CNS disorder \\
\hline $348.2,348.4,348.5,348.8,348.9$ & 348 other brain conditions \\
\hline
\end{tabular}

$A D=$ Alzheimer disease; $A L S=$ amyotrophic lateral sclerosis; $C N S=$ central nervous system; $C V A=$ cerebrovascular accident; EPS=extrapyramidal symptoms; ICD-9-CM=International Classification of Diseases, Ninth Revision, Clinical Modification; MEPS=Medical Expenditure Panel Survey; N/A=not applicable; $N E C=$ not elsewhere classified; $O A=$ osteoarthritis; $P D=$ Parkinson disease; $R A=$ rheumatoid arthritis; $S L E=$ systemic lupus erythematosus. 


\section{APPENDIX B Variables Used in the Analysis}

MEPS Variable Name

Description

Test or Regression Type Used for Significance

Descriptive variables

\begin{tabular}{l|l}
\hline AGEa $^{a}$ & A \\
\hline SEX $^{a}$ & \\
\hline RACEX $^{a}$ & \\
\hline EDUCYR $^{a}$ & \\
\hline INSCOV & \\
\hline WAGEPa & \\
\hline EMPST $^{a}$ &
\end{tabular}

\begin{tabular}{|l|l}
\hline Age & \\
\hline Gender & \\
\hline Race/ethnicity & Ch \\
\hline Years of education & Ch \\
\hline Insurance (public/private/uninsured) & \\
\hline Wage and salary income & Employment status
\end{tabular}

t-test

Chi-square test

Chi-square test

Chi-square test

Chi-square test

EMPST $^{a}$

Employment status

t-test

Disability variables

$\mathrm{DFCOG}^{\mathrm{a}}$

Serious cognitive difficulty

DFWLKC $^{a}$

Difficulty walking or climbing stairs

Chi-square test

Health-related quality of life variables

\begin{tabular}{|c|c|c|}
\hline PCS & PCS (physical) score of SF-12 & Linear \\
\hline MCSb & MCS (mental) score of SF-12 & Linear \\
\hline \multicolumn{3}{|c|}{ Health care resource use variables } \\
\hline OBTOTVb & Office-based provider visits & Negative binomial \\
\hline OPTOTVb & Outpatient-based provider visits & Negative binomial \\
\hline ERTOTb & Emergency room visits & Negative binomial \\
\hline IPDIS $^{b}$ & Inpatient hospital days & Negative binomial \\
\hline HHTOTD $^{\mathrm{b}}$ & Home health provider days & Poisson (lack of convergence with negative binomial) \\
\hline RXTOTb & Prescription fills & negative binomial \\
\hline \multicolumn{3}{|c|}{ Expenditure variables } \\
\hline OBVEXPb & Office-based provider expenditures & Extended estimating equations with recycled predictions \\
\hline OPVEXPb & Outpatient-based provider expenditures & $\begin{array}{l}\text { Two-part model: } \\
\text { 1. Logit } \\
\text { 2. Log-link gamma generalized linear model }\end{array}$ \\
\hline ERTEXPb & Emergency room expenditures & Extended estimating equations with recycled predictions \\
\hline IPTEXPb & Inpatient expenditures & Extended estimating equations with recycled predictions \\
\hline HHTEXPb & Home health expenditures & Extended estimating equations with recycled predictions \\
\hline RXEXPb & Prescription drug expenditures & Extended estimating equations with recycled predictions \\
\hline
\end{tabular}

